\title{
Leiomioma vulvar em lobo-guará (Chrysocyon brachyurus) - relato de caso
}

[Leiomyoma vulvar in maned wolf(Chrysocyon brachyurus) - case report]

G.A. Iglesias, T.L.C. Costa, T.O. Morgado, J.V.A. Galceran,

J.L.Q. Silva Júnior, H.J. Bento, M.A. Bianchini, K.S. Bezerra, C.A. Pescador, S.H.R. Corrêa

Universidade Federal de Mato Grosso - Hospital Veterinário - Cuiabá, MT

\section{RESUMO}

Descrição de leiomioma vulvar em lobo-guará (Chrysocyon brachyurus), fêmea, castrada, apresentando 10 anos de idade e mantida em cativeiro. Com base na avaliação clínica, no monitoramento de formação nodular de crescimento lento e progressivo e no diagnóstico preliminar sugestivo de neoplasia, procedeuse à exérese da massa tumoral e à identificação anatomopatológica do leiomioma. Exames complementares radiográficos e ultrassonográficos não apontaram presença de metástases. A ressecção cirúrgica se mostrou satisfatória como conduta clínica, não havendo recidiva.

Palavras-chave: canídeos selvagens, neoplasia

\begin{abstract}
Description of leiomyoma vulvar in a female castrated Chrysocyon brachyurus, , ten years of age, and maintained in captivity. Based on the clinical evaluation, monitoring of nodular formation of slow and progressive growth, and preliminary diagnosis suggestive of neoplasia, the tumor mass was excised and anatomopathological identification of the leiomyoma was done. Complementary radiographic and ultrasound examinations did not indicate the presence of metastases. Surgical resection proved to be satisfactory as a clinical practice, and there was no relapse.
\end{abstract}

Keywords: wild canids, neoplasia

\section{INTRODUÇÃO}

O lobo-guará (Chrysocyon brachyurus) pertence à ordem carnívora, família Canidae, e habita campos e cerrados da América do Sul (Paula e DeMatteo, 2015). Seu estado de conservação no mundo está classificado como "quase ameaçado",devido à perda de seu habitat, a atropelamentos nas rodovias e a conflitos com produtores rurais (Paula e DeMatteo, 2015; Maia e Gouveia, 2002). Apesar dos esforços conservacionistas direcionados a essa espécie, o decréscimo de sua população, especialmente em cativeiro, tem sido associado à incidência de doenças com etiologias variadas. Destacam-se afecções parasitárias pelo Dioctophyma renale, síndromes metabólicas, como a cistinúria e a urolitíase, afecções dentárias com presença de

Recebido em 2 de julho de 2017

Aceito em 28 de dezembro de 2018

E-mail: gabriella.a.iglesias@gmail.com hiperplasias gengivais severas, susceptibilidade ao parvovírus canino (Maia e Gouveia, 2002), além de afecções neoplásicas como aquelas relacionadas a tumores ovarianos (Munson e Montali, 1991) e carcinoma túbulo-papilar da glândula mamária (Gamba et al., 2011).

Os leiomiomas são considerados tumores benignos de células de músculo liso, não encapsulados e não invasivos, caracterizados por homogeneidade de células fusiformes densas, com bordas citoplasmáticas indistinguíveis e alongadas, núcleos de extremidades lisas dispostas em feixes entrelaçados, lembrando o tecido muscular liso normal (Cooper e Valentine, 2002).

Nos animais domésticos, os leiomiomas são encontrados no trato gastrointestinal e em vesícula biliar, assim como no sistema urinário 
de cães e gatos. Raramente são encontrados em outras espécies domésticas e silvestres e, quando ocorrem, acometem principalmente a vesícula urinária. Também podem ocorrer no baço, fígado, trato respiratório e, com menor frequência, em outros órgãos de músculo liso. Geralmente são achados de necropsia (Cooper e Valentine, 2002).

A incidência de leiomiomas em tecidos genitais é relatada com frequência em fêmeas, ocorrendo mais comumente naquelas não castradas e idosas, sendo essa neoplasia de base hormonal. São descritos no útero, na cérvix, na vagina e na vulva, podendo afetar a função reprodutiva. Os tumores mais volumosos podem causar obstrução de vias urinárias e de cólon. Os sinais clínicos mais comuns dos leiomiomas vaginais, vulvar e da cérvix são a presença de secreção vaginal e protrusão de massa vulvar (Cooper e Valentine, 2002).

Em felinos selvagens, o leiomioma uterino foi relatado em uma jaguatirica (Leopardus pardalis) fêmea, com aumento do volume abdominal, e em um leopardo (Panthera pardus) fêmea, que apresentou obstipação devido à compressão do cólon distal pela massa; esses animais foram submetidos a procedimento de remoção cirúrgica e avaliação anatomopatológica da massa tumoral, porém ambos vieram a óbito devido a complicações no pós-operatório (Gonçalves e Oliveira, 2000; Siegal-Willott et al., 2005).

Em um estudo post mortem realizado entre 1975 e 1995, foram analisados aproximadamente 30.000 casos de mamíferos exóticos pertencentes ao Zoo Biology and Wildlife Research (IZW), Berlin, e Smithsonian Institution, Washington DC, e o leiomioma foi descrito em útero, colo do útero e vagina de 14 espécies de mamíferos exóticos, incluindo o relato de uma fêmea de lobo-guará $(C$. brachyurus) com um tumor solitário na vagina (Hildebrandt et al., 1995). Em aves silvestres, o leiomioma já foi relatado, ocorrendo no pâncreas e no ligamento ventral do oviduto de columbiformes, no baço e no inglúvio de periquitos australianos (Sinhorini, 2008).

O tratamento do leiomioma preconiza a remoção da formação neoplásica, realizada na maioria dos casos por ressecção local, concomitante à ováriohisterectomia $(\mathrm{OVH})$ em fêmeas não castradas.
Em fêmeas já castradas, procede-se apenas à remoção da formação tumoral. Nos casos em que não existem metástases, o prognóstico para leiomiomas pode ser favorável (Menegassi et al., 2016). O objetivo do presente trabalho é relatar a presença de leiomioma vulvar em uma fêmea de lobo-guará, adulta, mantida em cativeiro no Zoológico da Universidade Federal de Mato Grosso, município de Cuiabá, estado de Mato Grosso, Brasil.

\section{CASUÍSTICA}

Foi encaminhada ao Hospital Veterinário da Universidade Federal de Mato Grosso (HovetUFMT) uma exemplar fêmea de lobo-guará $(C$. brachyurus), com idade estimada de 10 anos, pesando $23 \mathrm{~kg}$, multípara, castrada, mantida no Zoológico da Universidade Federal de Mato Grosso (Zoo-UFMT). O animal apresentava formação nodular, com cerca de três centímetros de diâmetro, no lábio vulvar direito, com crescimento lento e progressivo. O nódulo foi observado há cerca de dois anos após o animal ter sido submetido a procedimento cirúrgico, no qual se procederam à nefrectomia do rim direito devido ao parasitismo por Dioctophyma renale, e à ovário-histerectomia $(\mathrm{OVH})$ eletiva. Após o procedimento de $\mathrm{OVH}$, o útero foi analisado quanto as suas características histológicas e não apresentou nenhuma alteração. A partir de sua detecção, a formação nodular foi monitorada e investigada mediante punção por agulha fina (PAF) e realização de um imprinting em lâmina. A análise microscópica do material colhido demonstrou a presença de grande quantidade de hemácias e neutrófilos ocasionais, moderada quantidade de núcleos celulares disformes ou alongados e debrís celulares. Esse resultado, no entanto, não permitiu a confirmação diagnóstica da neoformação.

Diante do quadro clínico de evolução, optou se pela ressecção cirúrgica da formação nodular. Por meio de contenção física, com uso de um puçá, efetuou-se a aplicação do protocolo anestésico composto de cetamina $(8 \mathrm{mg} / \mathrm{kg})$ e midazolam $(0,5 \mathrm{mg} / \mathrm{kg})$, por via intramuscular. Em centro cirúrgico, efetuou-se a indução com propofol intravenoso $(5 \mathrm{mg} / \mathrm{kg})$, administrado a efeito, seguido de entubação orotraqueal com sonda orotraqueal número 9,5 para manutenção anestésica com isoflurano na concentração 
alveolar mínima (CAM) 1,3V\%, e posterior aplicação local de lidocaína $2 \%(8 \mathrm{mg} / \mathrm{kg})$.

A exérese da formação nodular foi realizada por divulsão da base da neoplasia, seguida de dermorrafia com fio de náilon 2.0-3.0. Após o procedimento cirúrgico, o animal foi submetido a exames radiográfico e ultrassonográfico para a certificação de que não havia metástases tumorais em órgãos internos. A recuperação póscirúrgica e a continuidade do tratamento foram realizadas com o animal novamente em seu recinto no Zoo da UFMT. Após dois anos de alta clínica, não foi observada recidiva de qualquer nova formação na região vulvar.

Macroscopicamente, o nódulo apresentava coloração branca, consistência firme, não encapsulado e com superfície de corte irregular. Então, foi armazenado em formol $10 \%$ e

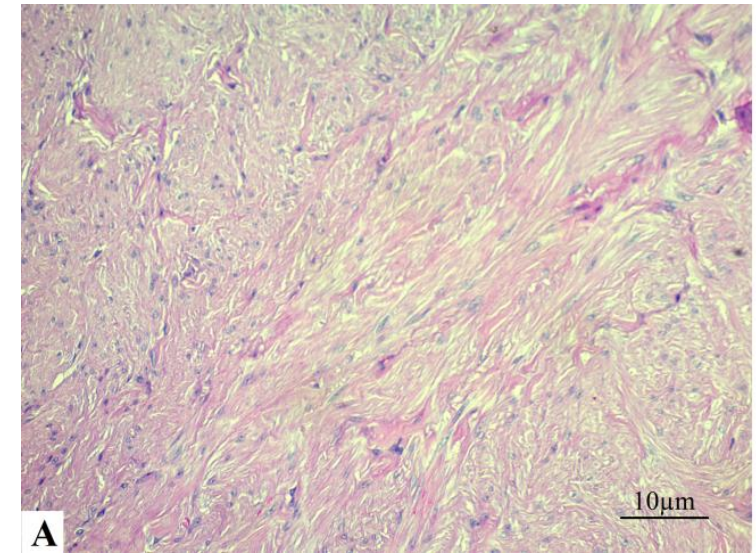

encaminhado ao Laboratório de Patologia Veterinária do Hovet-UFMT, sendo processado pelas técnicas convencionais de preparados histológicos permanentes, corado pelo método de hematoxilina e eosina (HE) e posteriormente avaliado em microscópio óptico.

Microscopicamente foi observada, na mucosa vulvar, uma formação nodular, moderadamente celular, composta por células fusiformes que se entrelaçavam em um padrão de paliçada. Essas células tinham bordos indistintos, moderada quantidade de citoplasma eosinofílico, fibrilar e, por vezes, vacuolizado. Os núcleos variavam de ovais a alongados, cromatina finamente pontilhada e um nucléolo evidente. Havia discreta anisocitose e anisocariose, e não foram observadas figuras de mitose (Fig. 1). Com base nos achados morfológicos, concluiu-se que o nódulo era compatível com leiomioma vulvar.

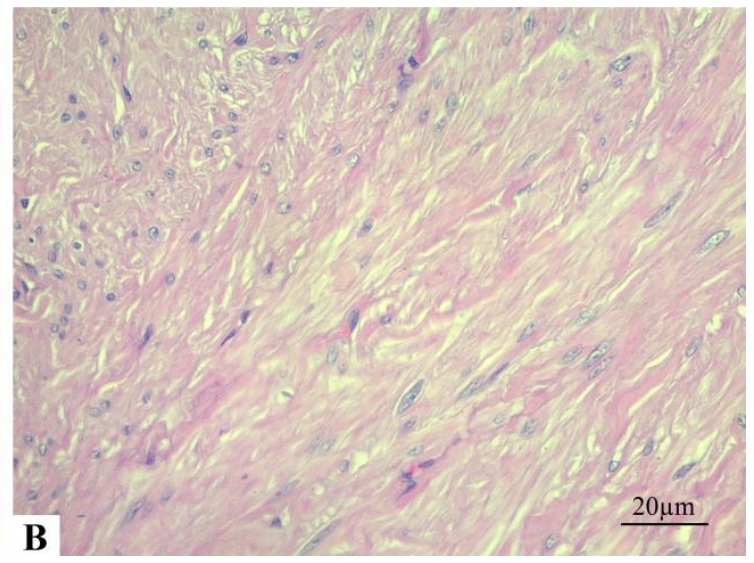

Figura 1. Leiomioma vulvar em lobo-guará (Chrysocyon brachyurus). Observa-se um padrão celular neoplásico uniforme, sem atipia, organizando-se em espirais ou faixas longitudinais com aspecto de musculatura lisa. Hematoxilina e eosina (HE), obj. 20x (A) e obj. 40x (B).

Posteriormente, a análise imuno-histoquímica (Fig. 2) foi realizada utilizando-se cortes de $3 \mu \mathrm{m}$ de tecidos fixados em formol e incluídos em parafina. Estes foram submetidos ao método da biotina-estreptavidina, utilizando-se o anticorpo actina de músculo liso. O bloqueio da peroxidase endógena foi realizado com solução de peróxido de hidrogênio (3\%), durante 15 minutos, em temperatura ambiente. A recuperação do antígeno foi feita pelo método do micro-ondas, incubando as lâminas durante três minutos em solução EDTA pH 9,0, em potência máxima. Em seguida, realizou-se lavagem e adição de proteinase $\mathrm{K}$ no tecido durante dois minutos. Para reduzir as reações inespecíficas, houve o bloqueio com caseína (leite em pó desnatado) a $5 \%$, por 30 minutos, em temperatura ambiente. A revelação foi realizada com cromógeno diaminobenzidina, por um período de 30 segundos, e, posteriormente, este foi contracorado com hematoxilina de Mayer. 


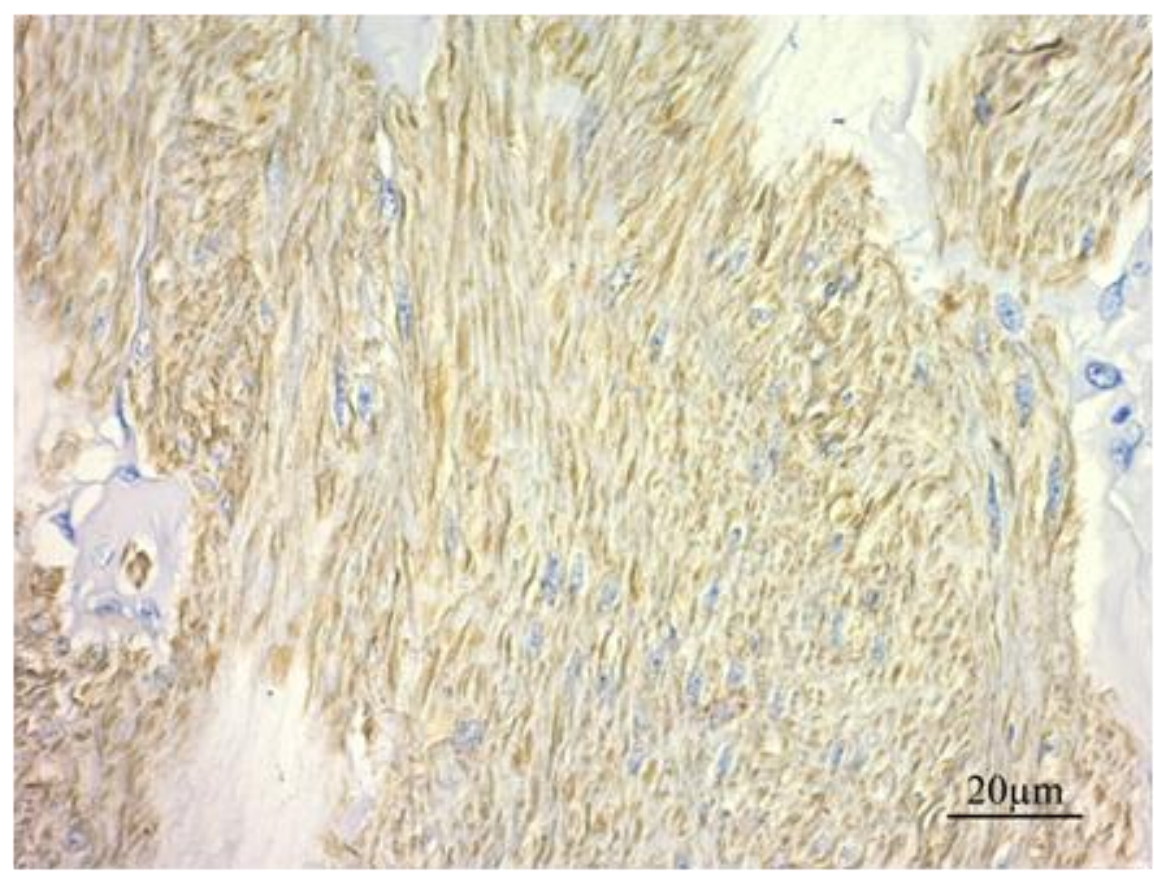

Figura 2. Expressão da actina de músculo liso pelas células musculares neoplásicas de leiomioma vulvar em lobo-guará (Chrysocyon brachyurus) (imuno-histoquímica, biotina-estreptavidina - obj. 40x).

\section{DISCUSSÃO}

O diagnóstico de leiomioma do nódulo vulvar da fêmea de lobo-guará ( $C$. brachyurus) avaliada neste relato foi baseado nas características macroscópicas, em que o nódulo apresentava coloração branca, consistência firme, não encapsulado e com superfície de corte irregular, além de seu crescimento lento e progressivo (Cooper e Valentine, 2002). As características histopatológicas mostraram-se também compatíveis com o relato de Menegassi et al. (2016), em sete cães fêmeas, que apresentavam células fusiformes de tamanho e formato uniforme, semelhantes ao tecido muscular liso, citoplasma indistinto e eosinofílico, com núcleo alongado deslocado perifericamente, e com coloração fortemente basofílica, demonstrando cromatina evidente. As células neoplásicas distribuíam-se em feixes intercalados por áreas de músculo liso normal, onde não eram observadas figuras de mitose.

A descrição histológica do tumor encontrado neste relato afastou a possibilidade da presença de um leiomiossarcoma. Histologicamente as características encontradas em leiomiossarcomas são muito semelhantes às de tumores solitários de leiomioma, porém o leiomiossarcoma é encontrado com maior frequência em fibras ou em componentes miofibroblásticos. Outra diferença encontrada entre esses tumores é que o leiomiossarcorma tem a capacidade de realizar metástase em outros órgaos, enquanto o leiomioma não o faz, sendo menos invasivo. A característica invasiva do leiomiossarcoma faz com que ocorra necrose tumoral, bem como a presença de células atípicas nessas áreas (Cooper e Valentine, 2002), características essas não visualizadas nas secções histológicas analisadas do presente caso.

Tumores de base hormonal similares podem ocorrer na musculatura lisa do trato reprodutivo de fêmeas, devendo o leimioma ser diferenciado desses tumores (Cooper e Valentine, 2002). A literatura não esclarece o papel do estrogênio na etiologia do leiomioma, acreditando-se que cães fêmeas que foram castrados tardiamente e apresentaram a neoplasia já haviam sofrido influência hormonal suficiente para o desenvolvimento dele, não havendo correlação entre a ocorrência da neoplasia vaginal e a idade em que a paciente foi castrada (Withrow e Susaneck, 1986). O mesmo fato foi observado no presente estudo, visto que a castração foi realizada de forma tardia, havendo a ocorrência do tumor mesmo após o procedimento. 
Alterações como cistos foliculares ovarianos, tumores secretores de estrógenos, hiperplasia endometrial, hiperplasia e neoplasia mamária podem estar frequentemente associados a desenvolvimento do leiomioma, segundo Schlafer e Mille (2007), porém a associação de afecções concomitantes dessa natureza não foi observada no animal do estudo em questão, visto que o útero apresentou normalidade histológica. Segundo Menegassi et al. (2016), a episiotomia é a técnica recomendada para remoção de formações nodulares intravaginais, no entanto, neste relato, a neoformação apresentava-se no lábio vulvar com proporção pequena de envolvimento de tecido perineal, sendo adotada a técnica de exérese de nódulo.

\section{CONCLUSÃO}

No presente relato, conclui-se que o lobo-guará (C. Brachyurus) é susceptível ao leiomioma vulvar. O procedimento cirúrgico de exérese da formação nodular mostrou-se efetivo, visto que, dois anos após a alta clínica do animal, não foram observadas novas formações nodulares, garantindo uma sobrevida e consequente melhora da qualidade de vida do paciente.

\section{REFERÊNCIAS}

COOPER, B.J.; VALENTINE, B.A. Tumors of Muscle. In: MEUTEN, D.J. (Ed.). Tumors in domestic animals. Iowa: United States of America, 2002. p.319-363.

GAMBA, C.O.; DAMASCENO, K.A.; FERREIRA, E. et al. Carcinoma túbulo-papilar da glândula mamária em lobo guará fêmea (Chrysocyon brachyurus): análise histopatológica e imunofenotípica. Arq. Bras. Med. Vet. Zootec., v.63, p.1377-1381, 2011.

GONÇALVES, G.F.; OLIVEIRA, S.T. Leiomioma uterino em jaguatirica (Leopardus pardalis) relato de caso. Arq. Ciênc. Vet. Zool. UNIPAR, v.3, p.185-188, 2000.

HILDEBRANDT T. B.; IPPEN, R.; KAISER, H. E. et al. Leiomyomas in the genital tract of captive exotic mammals. Anticancer Res., v.15, p.1754. 1995.
MAIA, O.B.; GOUVEIA, A.M.G. Birth and mortality of maned wolves Chrysocyon brachyurus (Illiger, 1811) in captivity. Braz. J. Biol., v.62, p.25-32, 2002.

MENEGASSI, C.C.; MARTINS, I.C.S.; PEREIRA, G.M. et al. Aspectos clínicos, cirúrgicos, histológicos e pós-operatórios de oito cadelas com leiomioma vaginal. Arq. Bras. Med. Vet. Zootec., v.68, p.307-312, 2016.

MUNSON, L.; MONTALI, R.J. High prevalence of ovarian tumors in maned wolves (Chrysocyon brachyurus). J. Zoo Wildl. Med., v.22, p.125129, 1991.

PAULA, R.C.; DEMATTEO, K. Chrysocyon brachyurus. (errata version published in 2016). The IUCN red list of threatened species 2015. Available in: <http://www.iucnredlist.org/details/4819/0>. Accessed in: 16 Feb. 2017.

SCHLAFER, D.H.; MILLER, R.B. Female genital system. In: MAXIE M.G. (Ed.). Jubb, Kennedy \& Palmer's pathology of domestic animals. Philadelphia: Elsevier, 2007. p.429-564.

SIEGAL-WILLOTT, J.L.; HENRIKSON, T.; CARPENTER, J.W. et al. Chronic obstipation in a leopard (Panthera pardus) caused by intrapelvic uterine leiomyoma compression of the distal colon. J. Zoo Wildl. Med., v.36, p.534$537,2005$.

SINHORINI, J.A. Neoplasias em aves domésticas e silvestres mantidas em domicílio: avaliação anatomopatológica e imunoistoquímica. 2008. 131f. Dissertação (Mestrado em Ciências) - Faculdade de Medicina Veterinária e Zootecnia, Universidade de São Paulo, São Paulo, SP.

WITHROW S.J.; SUSANECK, S.J. Tumor of the canine female reproductive tract. In: MORROW, D.A. (Ed.). Current therapy in theriogenology: diagnosis, treatment, and prevention of reproductive diseases in small \& large animals. Philadelphia: Saunders, 1986. p.521-528. 